\title{
On the interaction of microquasar jets with stellar winds
}

\author{
M. Perucho ${ }^{1}$ and V. Bosch-Ramon ${ }^{2}$
}

\author{
1 Max-Planck-Institut für Radioastronomie, Auf dem Hügel 69, Bonn 53121, Germany \\ e-mail: perucho@mpifr-bonn.mpg.de \\ 2 Max Planck Institut für Kernphysik, Saupfercheckweg 1, Heidelberg 69117, Germany \\ e-mail: vbosch@mpi-hd.mpg.de
}

Received 26 October 2007 / Accepted 6 February 2008

\section{ABSTRACT}

\begin{abstract}
Context. Strong interactions between jets and stellar winds at binary-system, spatial-scales could occur in high-mass microquasars. Aims. We study here, mainly from a dynamical but also a radiative point of view, the collision between a dense stellar wind and a mildly relativistic hydrodynamical jet of supersonic nature.

Methods. We have performed numerical two-dimensional simulations of jets, with cylindrical and planar (slab) symmetry, crossing the stellar-wind material. From the results of the simulations, we derive estimates of the particle acceleration efficiency, using firstorder, Fermi-acceleration theory, and provide insight into the possible radiative outcomes.

Results. We find that, during jet launching, the jet head generates a strong shock in the wind. During and after this process, strong recollimation shocks can occur due to the initial overpressure of the jet with its environment. The conditions in all these shocks are convenient to accelerate particles up to $\sim \mathrm{TeV}$ energies, which can lead to leptonic (synchrotron and inverse Compton) and hadronic (proton-proton) radiation. In principle, the cylindrical jet simulations show that the jet is stable, and can escape from the system even at relatively low power. However, when accounting for the wind ram pressure, the jet can be bent and disrupted for power $\lesssim 10^{36} \mathrm{erg} \mathrm{s}^{-1}$.
\end{abstract}

Key words. X-rays: binaries - stars: individual: LS 5039 - radiation mechanisms: non-thermal

\section{Introduction}

Strong shocks can take place in very different astrophysical scenarios, for example, due to the formation of structures in extragalactic galaxy clusters (e.g. Kang, et al. 1996), active galactic nuclei (AGN) jets (e.g. Begelman et al. 1984), supernova remnants (e.g. van der Laan 1962), star-forming regions (e.g. Bykov \& Fleishman 1992), pulsar winds (e.g. Rees \& Gunn 1974), galactic, relativistic jets (e.g. Zealey, et al. 1980), or jets in young stars (e.g. Araudo et al. 2007 and references therein).

In the case of AGNs, the interaction of jets with their environment allows the study of jet properties, such as matter content and energy budget, in the case of both Fanaroff-Riley II (FRII; e.g. Scheck et al. 2002; Krause 2005) and Fanaroff-Riley I (FRI; e.g. Perucho \& Martí 2007) sources. The simulation of extragalactic jets, and their interaction with the environment have been addressed by a number of authors during the past decades (e.g., Martí et al. 1997; Komissarov \& Falle 1998; Aloy et al. 1999; Rosen et al. 1999; Scheck et al. 2002; Leismann et al. 2005; Perucho et al. 2005, 2006; and Mizuno et al. 2007). In particular, the evolution of jets in homogeneous and inhomogeneous ambient media, the influence of internal energy on the structure of the jets, the mass load of external material, the evolution of FRI and FRII sources, and the growth of the Kelvin-Helmholtz instability in relativistic flows, have all been thoroughly studied.

In the case of microquasars, X-ray binary systems with relativistic jets (e.g. Mirabel \& Rodríguez 1999; Ribó 2005), there have not been many studies of the consequences of the interaction between the jet and the surrounding medium (e.g. Velázquez \& Raga 2000). A common approach has been to compare with extragalactic jets to point out similarities between the two types of sources (e.g., Gómez 2001; Hardee \& Hughes 2003). It appears clear that these galactic outflows should generate some kind of structure in their termination region, although the characteristics of this region from the radiative and dynamical point of view are unclear (e.g. Aharonian \& Atoyan 1998; Heinz 2002; Heinz \& Sunyaev 2002; Bosch-Ramon et al. 2005; Bordas et al., in preparation), and only a handful of sources show hints or evidences of jet/medium interaction (e.g. SS 433, Zealey et al. 1980; XTE J1550-564, Corbel et al. 2002; Cygnus X-3, Heindl et al. 2003; Cygnus X-1, Martí et al. 1996; Gallo et al. 2005; H1743-322, Corbel et al. 2005; Circinus X-1, Tudose et al. 2006; LS I +61 303, Paredes et al. 2007).

A microquasar jet can interact strongly not only with the interstellar medium. In high-mass microquasars (HMMQ), the massive and hot primary star suffers severe mass loss in the form of a supersonic wind that can embed the jet ${ }^{1}$. This wind can have a strong impact on the jet dynamics on binary-system, spatialscales. The jet may be destroyed by the wind, preventing jet detection. In such a scenario, shocks are likely to occur, leading to efficient particle acceleration and the production of multiwavelength radiation. This illustrates the importance of studying the interaction between the jet and the stellar wind, using hydrodynamics. Previous works treated the jet/stellar wind interaction using a more phenomenological approach, and focused on

\footnotetext{
1 We focus on HMMQs due to the relevance of the stellar wind at the spatial scales studied here. The presence of dense material and the stellar photon field makes HMMQs even more interesting from the radiative point of view. In the case of low mass systems, there might be accretion disk winds affecting jet dynamics (e.g. Tsinganos et al. 2004), but the properties of the environment are not well known and the impact on the jet is less clear.
} 
possible radiative outcomes (e.g. Romero et al. 2003; Romero et al. 2005; Aharonian et al. 2006a). Regarding the production of non-thermal particles in the present context, diffusive shock (first-order Fermi) acceleration is a plausible mechanism (e.g. Drury 1983). Other mechanisms of particle acceleration may be operating as well, such as second-order Fermi acceleration (Fermi 1949), shear acceleration (e.g. Rieger \& Duffy 2004), or the so-called converter mechanism (e.g. Derishev et al. 2003).

In this work, we present the results from two-dimensional relativistic hydrodynamical simulations, with cylindrical and slab symmetry, in a simplified scenario of the jet/stellar wind interaction in an HMMQ. Our principal goal is to provide firstorder estimates of the dynamical impact of the wind on the jet. In addition to this, we provide semi-analytical estimates on the radiative outcome of the simulation results.

In the next section, we briefly describe the main features of the scenario considered and present the physical parameters of the simulations; in Sect. 3, we characterize the setup of the simulations and explain their technical aspects; in Sect. 4, we present the results obtained, which are discussed in Sect. 5, together with the consequences derived from the simulations, from the point of view of radiation or jet stability. We summarize results and conclusions in Sect. 6.

\section{Physical scenario}

The physical scenario adopted here corresponds to an HMMQ with physical parameters similar to those of Cygnus X-1 or LS 5039, two close high-mass X-ray binaries with jets and with moderate-to-strong stellar-mass loss from a primary $\mathrm{O}$ star, in the form of a fast and supersonic wind. The distance between the jet base and the primary star is fixed to $R_{\text {orb }}=3 \times 10^{12} \mathrm{~cm}$. This distance is similar to those in LS 5039 (Casares et al. 2005) and Cygnus X-1 (Gies \& Bolton 1986). Typical luminosities and photon energies for $\mathrm{O}$ stars are $L_{*} \approx 10^{39} \mathrm{erg} \mathrm{s}^{-1}$ and $\epsilon_{0} \approx 3 \mathrm{kT} \approx 10 \mathrm{eV}$, respectively; the stellar wind present speeds $V_{\mathrm{w}} \approx 2 \times 10^{8} \mathrm{~cm} \mathrm{~s}^{-1}$, mass loss rates $\dot{M}_{\mathrm{w}} \approx 10^{-6} M_{\odot} \mathrm{yr}^{-1}$, and temperatures $T_{\mathrm{w}} \sim 10^{4} \mathrm{~K}$.

For the jet, we adopt a hydrodynamical supersonic outflow, ejected perpendicular to the orbital plane, at mildly relativistic velocities, $V_{\mathrm{j}, 0} \sim 10^{10} \mathrm{~cm} \mathrm{~s}^{-1}$, and temperature $T_{\mathrm{j}} \approx 10^{9-10} \mathrm{~K} \ll$ $m_{\mathrm{p}} c^{2} / k_{\mathrm{B}}$, where $m_{\mathrm{p}}$ is the proton mass, $c$ is the speed of light and $k_{\mathrm{B}}$ is the Boltzmann constant. The choice of temperature is arbitrary because we only assume that the jet is supersonic. Nevertheless, the temperature plays a role and its relevance is discussed in Sect. 5. In this work, we focus on jets formed by protons and electrons, as expected in cases where the jet is fed with particles from the accretion disk. Given the adopted temperatures and jet speeds, the most reasonable choice of adiabatic exponent is $\Gamma=5 / 3$.

If we consider a leptonic jet, the adopted temperatures would imply an important increase in the internal energy of the particles. This would significantly reduce the Mach number, affecting the assumption of a supersonic flow used here. Scheck et al. (2002) have shown that a supersonic leptonic jet with the same energy flux than a hadronic jet, develops in a very similar way. Therefore, large differences are not expected if the kinetic luminosity of the jet remains constant, regardless of its composition.

Regarding the geometry adopted for the simulation, a cylindrical symmetry can suffice to study the jet head shock formation in the wind. Since the jet speed $V_{\mathrm{j}} \gg V_{\mathrm{w}}$, the wind speed (which breaks the cylindrical symmetry) is neglected. As it will be seen in the slab simulations, this assumption is reasonable for powerful jets, but not in the case of weaker ones. At the present stage, slab symmetry simulations are sufficient to show the importance of the stellar wind ram pressure on the jet, because the timescales on which the wind surrounds the jet $\left(\gtrsim R_{\mathrm{j}} / V_{\mathrm{w}}\right.$; where $R_{\mathrm{j}}$ is the jet radius) are longer than the simulation timescales. Also, the adopted configuration of the stellar wind, emanating from one side of the jet, is consistent with an orbital timescale much longer than the simulation timescales. These simplifications are reasonable in our case, because we plan to highlight remarkable features and not to obtain accurate quantitative predictions.

We note that the wind structure is an important point in our simulations. It has been proposed that massive stellar winds may be porous (e.g. Owocki \& Cohen 2006). In addition, mass-loss rates of $\mathrm{OB}$ stars may be smaller up to a factor of several, due to wind clumping, as shown, e.g., in Puls et al. (2006), where estimates for the real mass-loss rates of several $\mathrm{O}$ stars, after wind clumpiness correction, are provided. For extremely porous winds, the collision of the jet with small and dense clumps (i.e. the dynamically-relevant part of the wind) would be rare, although these interactions could have interesting radiative outcomes (e.g. Aharonian \& Atoyan 1996; Romero et al. 2007a). Smaller mass-loss rates would in addition weaken the jet kinetic luminosity constraints derived in this work.

In our simulations, the jet starts at a distance $z_{0}=6 \times 10^{10} \mathrm{~cm}$ from the compact object, where it should be still little affected dynamically by the environment. The initial half-opening angle of the jet is taken to be 0.1 radians, which is given by the relation between the advance and expansion velocities ${ }^{2}$. The jet then propagates through the system, sweeping up stellar-wind material. Two shocks are eventually generated, one in the stellar wind (i.e. a forward or bow shock), and another in the jet itself (i.e. a backwards or reverse shock). The shocked material is referred to as shell for the wind (i.e. a bow shock downstream), and cocoon for the jet (i.e. a reverse shock downstream). The jet continues to advance but slows down due to kinetic-energy exchange with swept-up wind material. It can eventually be bent, and even destroyed, by the stellar-wind ram pressure. The pressure of the wind, or cocoon, as compared to the lateral pressure of the jet, can give rise to strong recollimation shocks. Recollimation shocks can destroy the jet, via deceleration and loss of collimation of the fluid downstream of the shocks (Perucho \& Martí 2007).

\section{Jet/wind interaction simulations}

We performed five numerical simulations, three with cylindrical symmetry and two with slab symmetry, in order to study the evolution of HMMQ jets with different injection powers.

The simulations were performed using a two-dimensional finite-difference code based on a high-resolution shockcapturing scheme, which solves the equations of relativistic hydrodynamics written in conservation form. This code is an upgrade of the code described in Martí et al. (1997) (e.g. see Perucho et al. 2005). Simulations were performed in two dualcore processors in the Max-Planck-Institut für Radioastronomie.

The numerical grid of the cylindrical-geometry simulations is formed by 320 cells in the radial direction and 2400 cells in the

\footnotetext{
2 A jet in free expansion, due to a large initial overpressure, expands approximately at its sound speed (Leahy 1991), which is, in our simulations, roughly an order of magnitude smaller than the advance speed (for the temperatures given in Sect. 3), and results in the opening angle indicated above.
} 
Table 1. Parameters of the wind.

\begin{tabular}{ll}
\hline \hline Parameter & \\
\hline Wind density $\left(\mathrm{g} \mathrm{cm}^{-3}\right)$ & $2.8 \times 10^{-15}$ \\
Wind pressure $\left(\mathrm{erg} \mathrm{cm}^{-3}\right)$ & $1.5 \times 10^{-3}$ \\
Wind velocity $\left(\mathrm{cm} \mathrm{s}^{-1}\right)$ & $2 \times 10^{8}$ \\
Wind mass-loss rate $\left(M_{\odot} \mathrm{yr}^{-1}\right)$ & $10^{-6}$ \\
Wind ram-pressure $\left(\mathrm{erg} \mathrm{cm}^{-3}\right)$ & $1.12 \times 10^{2}$ \\
\hline
\end{tabular}

Table 2. Parameters of the jets.

\begin{tabular}{llll}
\hline \hline Parameter & I & II & III \\
\hline Jet power $\left(\mathrm{erg} \mathrm{s}^{-1}\right)$ & $3.0 \times 10^{34}$ & $10^{36}$ & $3.0 \times 10^{37}$ \\
Jet init. pressure $\left(\mathrm{erg} \mathrm{cm}^{-3}\right)$ & 9.1 & 68 & $6.2 \times 10^{4}$ \\
Jet init. density $\left(\mathrm{g} \mathrm{cm}^{-3}\right)$ & $2.2 \times 10^{-16}$ & $5.9 \times 10^{-16}$ & $1.8 \times 10^{-14}$ \\
Jet init. temperature (K) & $7.4 \times 10^{8}$ & $2.1 \times 10^{9}$ & $6.2 \times 10^{10}$ \\
Jet init. speed $\left(\mathrm{cm} \mathrm{s}^{-1}\right)$ & $1.3 \times 10^{10}$ & $2.2 \times 10^{10}$ & $2.2 \times 10^{10}$ \\
Jet init. Mach number & 47.02 & 51.05 & 9.35 \\
\hline
\end{tabular}

axial direction in a uniform region, with physical dimensions of $20 \times 300 R_{\mathrm{j}}$. An expanded grid with 160 cells in the transversal direction, brings the boundary from $20 R_{\mathrm{j}}$ to $60 R_{\mathrm{j}}$, whereas an extended grid in the axial direction, consisting of 480 extra cells, spans the grid axially from $300 R_{\mathrm{j}}$ to $450 R_{\mathrm{j}}$. The enlargement of the grid is necessary to ensure that the boundary conditions are sufficiently far from the region of interest, and to avoid numerical reflection of waves in the boundaries affecting our results. The numerical resolution in the uniform grid is thus of 16 cells $/ R_{\mathrm{j}}$ in the radial direction and 8 cells $/ R_{\mathrm{j}}$ in the axial direction. Outflow boundary conditions are used on the outer boundaries of the grid, inflow at injection, and reflection at the jet axis in the cylindrical case. In the simulations, all the physical variables are scaled to the units of the code, which are the jet radius $R_{\mathrm{j}}$, the rest-mass density of the ambient medium $\rho_{\mathrm{a}}$, and the speed of light $c$.

The jet is injected in the grid at a distance of $6 \times 10^{10} \mathrm{~cm}$ from the compact object, and its initial radius is taken to be $R_{\mathrm{j}, 0}=6 \times 10^{9} \mathrm{~cm}$. The time unit of the code is thus equivalent to $0.2 \mathrm{~s}$, derived using the radius of the jet at injection and the speed of light $\left(R_{\mathrm{j}, 0} / c\right)$. The grid covers the distance between $6 \times 10^{10} \mathrm{~cm}$ and $2 \times 10^{12} \mathrm{~cm}$, i.e., $60 \%$ of $R_{\text {orb }}$. The ambient medium, i.e. the stellar wind, is composed of a gas with thermodynamical properties derived from Sect. 2 (see Table 1). Both the jet and the ambient medium are considered to be formed by a non-relativistic gas with adiabatic exponent $\Gamma=5 / 3$.

In the simulations, the jets are injected with different properties. The physical parameters that characterize the three simulations, are listed in Table 2. The main difference between simulations is the kinetic luminosity $\left(L_{\mathrm{j}}\right)$ : for a weak jet (case I) this is $3 \times 10^{34} \mathrm{erg} \mathrm{s}^{-1}$; for a mild jet (case II), it is $10^{36} \mathrm{erg} \mathrm{s}^{-1}$; and for a powerful jet (case III), $3 \times 10^{37} \mathrm{erg} \mathrm{s}^{-1}$. The selection of the jet power is addressed to show some illustrative cases: the lowest value, that of case I, is similar to the minimum power required to power radio emission under reasonable assumptions of the radio emitter (e.g. for Cygnus X-1, see Heinz 2006); that of case II is a bit smaller than the power estimates found for Cygnus X-1 (Gallo et al. 2005), and similar to the $L_{\mathrm{j}}$ inferred for LS 5039 (Paredes et al. 2006), and the maximum value, that of case III, is between the upper limit for the Cygnus X-1 $L_{\mathrm{j}}$ and the $L_{\mathrm{j}}$ of SS 433 (e.g. Gallo et al. 2005; Marshall et al. 2002). These jets are evolved until they have reached distances similar to the binary system size.
The velocities of the jets were selected as those of mildly relativistic flows, with Lorentz factors $\gamma_{j}=1.1$ for case I, and $\gamma_{\mathrm{j}}=1.5$ for cases II and III. The jet Mach numbers are similar in cases I (47.02) and II (51.05), whereas it is much smaller in case III (9.35). In the latter, the increase in the jet power is due to an increase in the internal energy of the jet, keeping the same jet speed as in case II. The jet temperatures also show this fact, because the jet in case III has a temperature larger than that of case II by a factor 30. This allows us to see the influence of changes of the velocity and the internal energy on the jet evolution.

In the case of the slab geometry simulations, the grid size is shortened to $200 R_{\mathrm{j}}$ along the jet axis, and it is doubled in the direction transversal to the jet axis (it extends from $-60 R_{\mathrm{j}}$ to $60 R_{\mathrm{j}}$ ). The numerical resolution is identical to that used in the cylindrical simulations. The jet is injected into an ambient medium that mimics the presence of a spherical wind, centered on a star at a distance $R_{\text {orb }}$ from the compact object, in the orbital plane, with the properties mentioned in Sect. 2. In this case, the boundary conditions are injection on the side from which the wind is assumed to originate and on the base of the jet, and outflow in the outer boundaries, on the opposite side of the grid and at the end of the grid in the axial direction. This kind of simulations was completed for cases $\mathrm{I}$ and $\mathrm{II}^{3}$, since the interaction with the wind was expected to be relevant to the jet dynamics.

\section{Results}

\subsection{Case l: a weak jet}

\section{Cylindrical jet}

The jet in case I, travels the distance between the injection and outer boundary of the numerical grid, in a time of $t_{\mathrm{f}} \simeq 900 \mathrm{~s}$. The bow shock generated by the injection of the jet in the ambient medium, moves at a mean speed of $V_{\mathrm{bs}} \sim 0.06 \mathrm{c}$. The jet generates a high-pressure cocoon that keeps it collimated (see the discussion in the next section). No additional shocks or significant jet perturbations are generated, and the jet propagates well collimated through the wind. We note that the speed of the unshocked jet does not change significantly along the axis. We do not show any image of this simulation due to the lack of remarkable features like shocks. A similar result was obtained in case II. We thus refer the reader to Fig. 2 (see Sect. 4.2).

Our approximation of a static wind is reviewed critically after results of the slab-jet simulation are presented below. In this simulation, the wind has the appropriate velocity.

\section{Slab jet}

A similar simulation was completed in planar coordinates, including the wind motion. The wind emanates from a point source, at the location of the primary star. Figure 1 shows panels of axial velocity, rest-mass density and pressure, at the moment that the jet head leaves the grid. The impact of the wind on the evolution of weak jets is clear from the plots. The bow shock generated by the jet moves at a speed of $0.05 c$, and is deformed by the wind and asymmetry in pressure on each side of the jet. The wind pushes the jet sideways, and two diagonal shocks, at $z \lesssim 3 \times 10^{11} \mathrm{~cm}$, are formed due to the interaction with the environment, which is in overpressure with respect to the jet flow. After the first shock, the jet widens and recollimates, starting a new process of expansion after $z \approx 2.6 \times 10^{11} \mathrm{~cm}$. The jet

\footnotetext{
3 The slab jets have the same lateral pressure and $L_{\mathrm{j}} / \mathrm{cm}-$ on the simulation plane - as in the cylindrical case.
} 

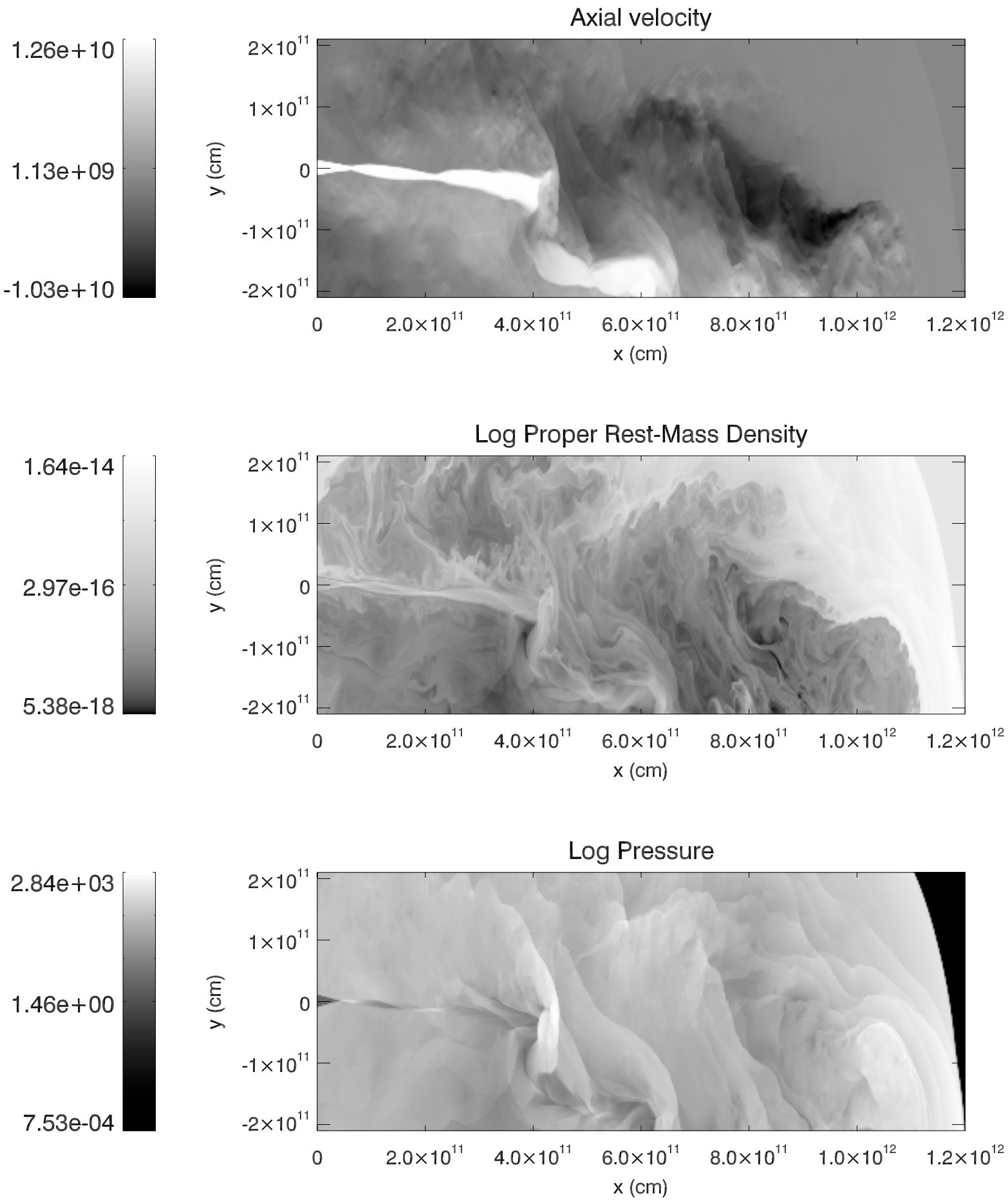

Fig. 1. Maps of axial velocity $\left(\mathrm{cm} \mathrm{s}^{-1}\right)$, rest mass density $\left(\mathrm{g} \mathrm{cm}^{-3}\right)$ and pressure $(\mathrm{dyn})$ at the end of the simulation $\left(t_{\mathrm{f}}=680 \mathrm{~s}\right)$ of a weak jet in slab geometry, case I. The horizontal and vertical coordinates indicate distances $(\mathrm{cm})$ to the injection point in the numerical grid and the jet axis, respectively. The stellar wind is centered on the position of the primary star, at $x=-6 \times 10^{10} \mathrm{~cm}$ and $y=3 \times 10^{12} \mathrm{~cm}$ in the figure.

is finally disrupted due to the action of the wind, after the jet has lost some of its initial inertia due to the previous internal shocks. A strong shock is generated at the disruption point at $z \approx 5 \times 10^{11} \mathrm{~cm}$. Beyond this point, the jet is diverted significantly, and some of the fast material, in the remaining outflow, leaves the regular numerical grid sideways at $z \sim 6-7 \times 10^{11} \mathrm{~cm}$. This simulation illustrates the case of a frustrated jet that has become a decollimated, slow outflow due to the effect of the wind.

\subsection{Case II: a mild jet}

\section{Cylindrical jet}

The simulation of case II in cylindrical coordinates is similar to that of case I. Figure 2 shows the rest-mass density and pressure of the jet, as its head reaches the end of the grid. Some internal shocks are produced by the slight overpressure of the jet, with respect to the cocoon at $z>1.4 \times 10^{12} \mathrm{~cm}$ : this is responsible for the main differences between the results for this case and of 
Log Proper Rest-Mass Density
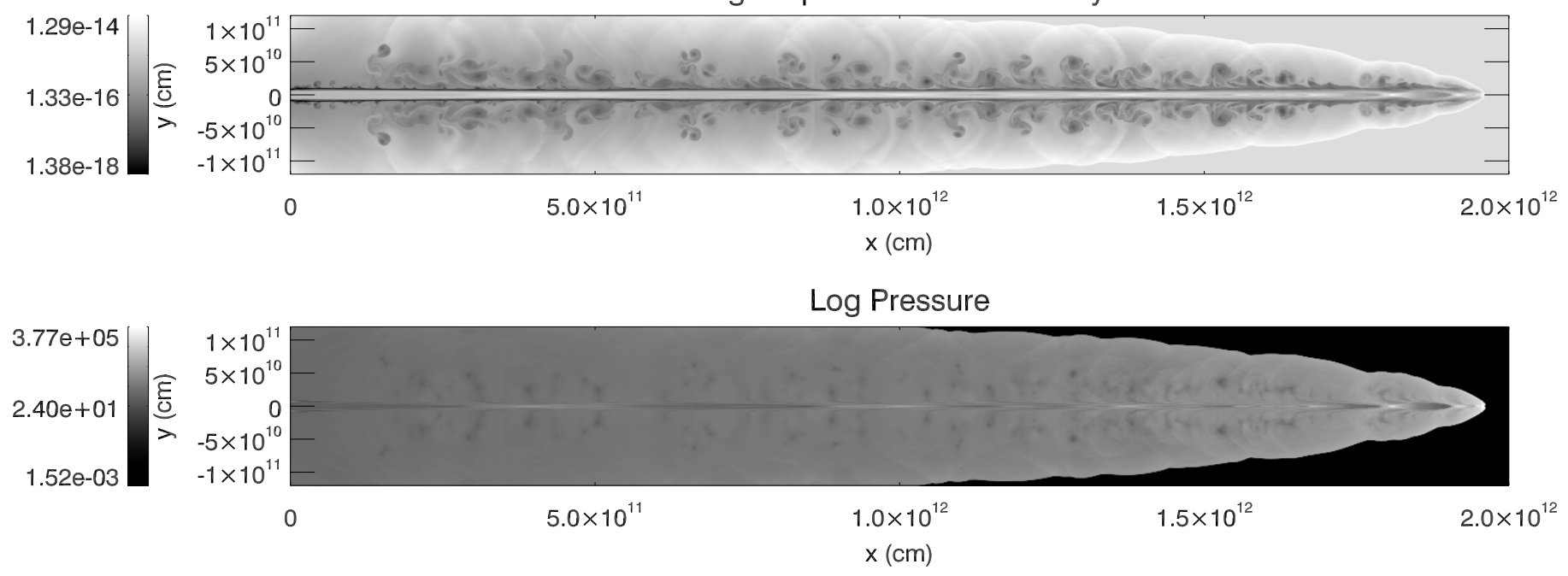

Fig. 2. Maps of rest mass density $\left(\mathrm{g} \mathrm{cm}^{-3}\right)$ and pressure (dyn) at the end of the simulation $\left(t_{\mathrm{f}}=164 \mathrm{~s}\right)$ of the mild jet (case II) in cylindric geometry. The horizontal and vertical coordinates, and the star location, are like in Fig. 1.

case I. Before $z \sim 1.4 \times 10^{12} \mathrm{~cm}$, the jet is collimated efficiently by the high-pressure cocoon. The bow-shock propagates at $V_{\mathrm{bs}} \sim 0.3 c$, and is therefore significantly faster than in the previous case. The bow-shock introduces a jump in pressure of $10^{8}$ with respect to the cold wind. The internal, conical shocks observed in the jet itself, show jumps in pressure of $10^{2}-10^{4}$, although there are no strong changes of axial velocity with respect to the unshocked jet material. This implies that not much kinetic energy could be transferred to non-thermal particles via these shocks.

\section{Slab jet}

In case II, a slab simulation was performed, accounting for the velocity of the stellar wind from the primary star. Figure 3 shows several maps of physical magnitudes from the simulation. The bow-shock is faster than for the slab case I $\left(V_{\mathrm{bs}} \sim 0.3 c\right)$. Several diagonal shocks are formed due to interaction with the wind, in the direction of the jet trajectory. The jet is in under pressure with respect to the ambient medium, which generates an initial collimation. The pinching triggered by the first collimation shock and the subsequent expansion of the jet, and the thrust exerted by the wind on the jet, together, generate nonlinear structures on the jet. In this case, the jet is finally disrupted at $z \sim 10^{12} \mathrm{~cm}$ (see Fig. 3). The pattern of shocks at the disruption region is similar to that observed in Fig. 1. These results show that the jet can be disrupted by the stellar-wind ram pressure even for intermediate kinetic luminosities.

\subsection{Case III: a powerful jet}

The simulation of a powerful jet $\left(L_{\mathrm{j}}=3 \times 10^{37} \mathrm{erg} \mathrm{s}^{-1}\right)$ produces results that are significantly different from those of the previous cylindrical simulations. Figure 4 displays maps of rest-mass density, pressure and axial velocity, as the bow-shock reaches the limit of the regular grid. The bow-shock propagates at $V_{\mathrm{bs}} \sim$ $0.3 c$. This $V_{\mathrm{bs}}$ is the same as in case II because part of the larger initial thrust of case III jet is invested in lateral expansion and heating. The latter happens due to, in contrast to the previous cases, a strong recollimation shock formed at $z \approx 1.3 \times 10^{12} \mathrm{~cm}$. This shock is formed due to the initial overpressure of the jet with respect to the cocoon.
The formation of such shocks is dependent on the relation between pressures in the cocoon and jet. The jump in pressure generated on the jet axis is of the order of $10^{4}$. In a second stage of jet evolution, when the bow-shock is away from the studied region and the cocoon is substituted by the stellar wind, recollimation shocks can change their position and properties. However, as is shown in the next section, these shocks may still be present. Such shocks, if sufficiently strong, can trigger pinching of the jet, as observed in Fig. 4, which, in turn, can lead to mass load of ambient gas and jet disruption (Perucho \& Martí 2007). This explains the importance of such recollimation shocks, not only in terms of particle acceleration and subsequent radiation, but also jet dynamics.

\section{Discussion}

\subsection{On the formation of shocks in the studied region}

We see that two different types of shock may form: reverse/bow shocks, which cross the region of constant density (within $\sim R_{\text {orb }}$ ) at a fraction of $c$ on timescales $<10^{4} \mathrm{~s}$; and recollimation shocks, generated by the interaction of the jet with its environment, the cocoon or the stellar wind itself. Unlike the reverse/bow shock, which appears naturally as shown by the simulations, the formation of recollimation shocks depends on the complex jet/medium pressure balance, and deserves further analysis. The possibility that they arise is discussed in this section in terms of the jet properties and their environments.

Recollimation shocks form when an initially-overpressured jet expands and reaches a lower pressure than that of its environment. In order to know whether a jet generates such a shock, and at which distance, the change of jet pressure with distance is needed to be compared with that of the ambient medium. In an adiabatic expansion, the condition $\rho_{\mathrm{j}} \gamma_{\mathrm{j}} V_{\mathrm{j}} A_{\mathrm{j}}=C_{1}$ holds, where $C_{1}$ is a constant and $A_{\mathrm{j}}$ is the jet cross section. In our simulations, the jet velocity and Lorentz factor are basically constant, implying $\rho_{\mathrm{j}} \propto 1 / R_{\mathrm{j}}^{2}$, which, for a conical jet, transforms into $\rho_{\mathrm{j}} \propto 1 / z^{2}$, i.e., the density of the jet decreases as the square of the distance to the source. It is also known that the jet pressure $\left(P_{\mathrm{j}}\right)$ and density satisfy the relation $P_{\mathrm{j}} / \rho_{\mathrm{j}}^{\Gamma}=C_{2}$, where $\Gamma$ is the 
$2.24 e+10$
$6.46 e+09$
$-9.52 e+09$

$1.71 \mathrm{e}-14$
$4.93 \mathrm{e}-16$
$1.42 \mathrm{e}-17$

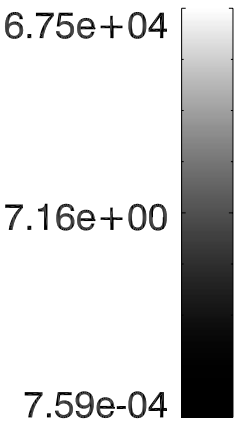

\section{Axial velocity}

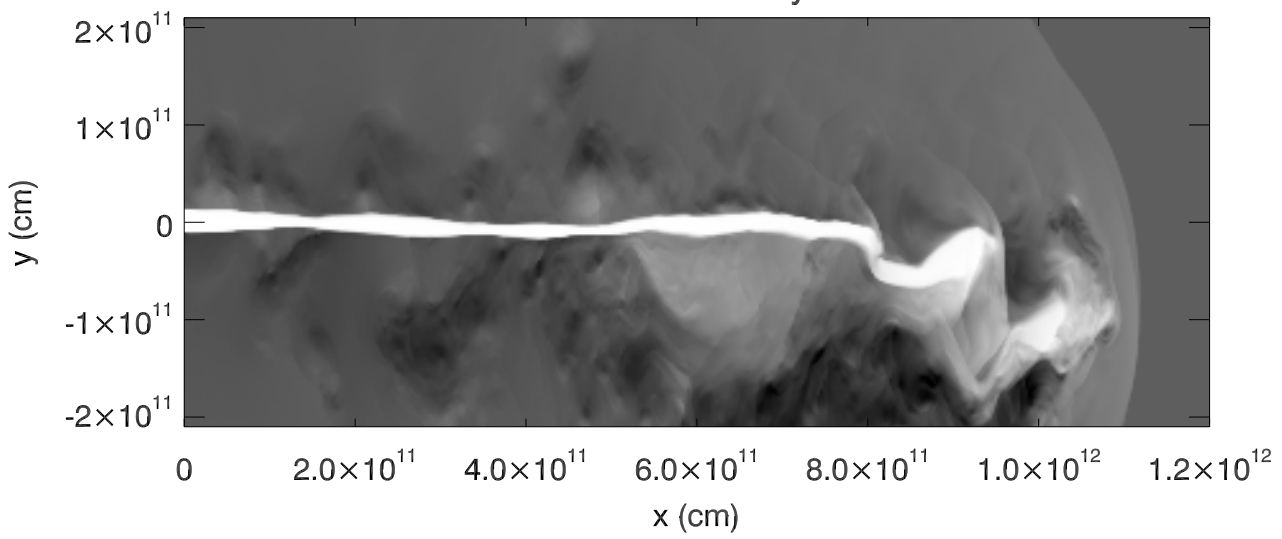

Log Proper Rest-Mass Density

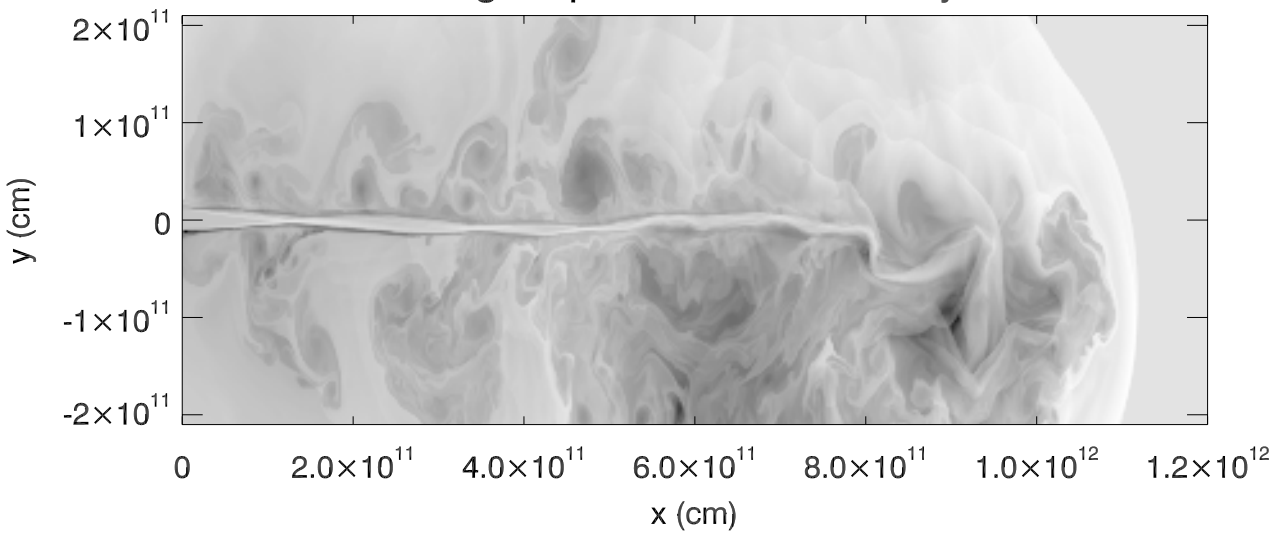

\section{Log Pressure}

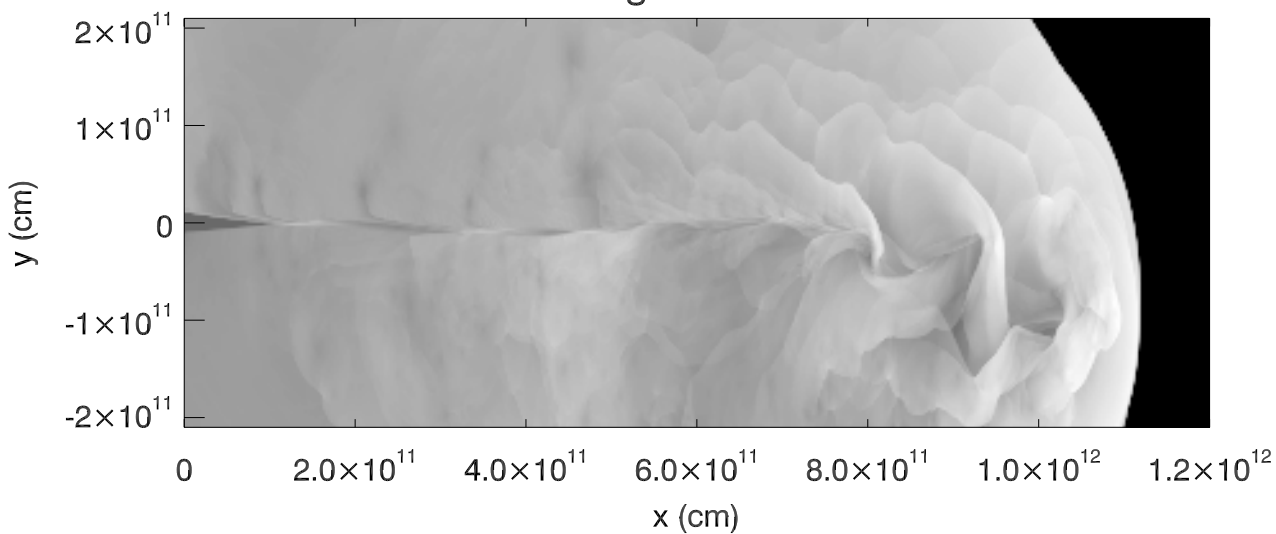

Fig. 3. Maps of axial velocity $\left(\mathrm{cm} \mathrm{s}^{-1}\right)$, rest mass density $\left(\mathrm{g} \mathrm{cm}^{-3}\right)$ and pressure $($ dyn $)$ at the end of the simulation $\left(t_{\mathrm{f}}=140 \mathrm{~s}\right)$ of a mild jet in slab geometry, case II. The horizontal and vertical coordinates, and the star location, are like in Fig. 1.

adiabatic exponent and $C_{2}$ a constant. Thus, $P_{\mathrm{j}} \propto 1 / z^{2 \Gamma}$, which implies $(\Gamma=5 / 3)$ :

$P \propto z^{-10 / 3}$.

A minimum distance for the position of a recollimation shock can be given as the place where the pressure in the jet is the same as the pressure of the ambient medium $\left(z_{\text {eq }}\right)^{4}$. We can express

\footnotetext{
${ }^{4}$ Actually, the shock occurs at a distance given by $z_{\mathrm{eq}}$, the Mach number of the jet at this point $(M)$, and the jet radius at $z_{\mathrm{eq}}\left(R_{\mathrm{eq}}\right)$ : $z \sim z_{\text {eq }}+R_{\text {eq }} \times M$ (see e.g. Leahy 1991), as the information takes a
}

this distance in terms of the pressure of the jet, at a reference position $z_{0}\left(P_{\mathrm{j}, 0}\right)$, which will be taken to be the injection point

certain time to reach the jet axis. At this distance, the pressure in the jet is strictly smaller than that of the ambient. In fact, the distance at which the curvature of the jet starts to change, which can be identified with the place where $P_{\mathrm{j}} \sim P_{\text {ext }}$, is about half of that in which the shock occurs (see Fig. 4). The second part of the equation depends on the parameters of the jet in the equilibrium point. For simplicity, we adopt the $z_{\text {eq }}$ as a lower-limit approximation to the position of the shock. 
Axial velocity

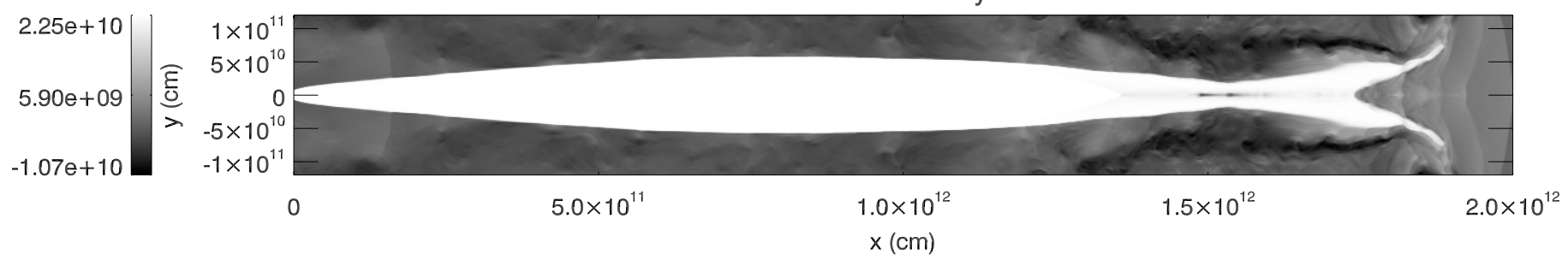

Log Proper Rest-Mass Density

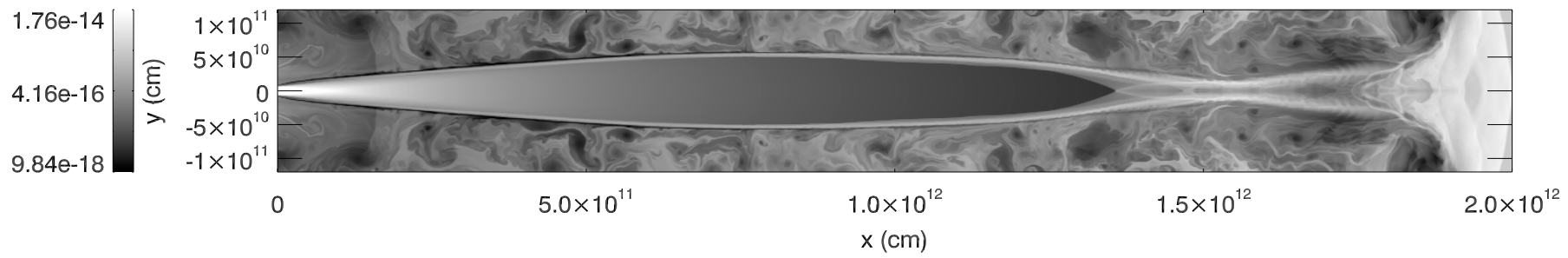

Log Pressure

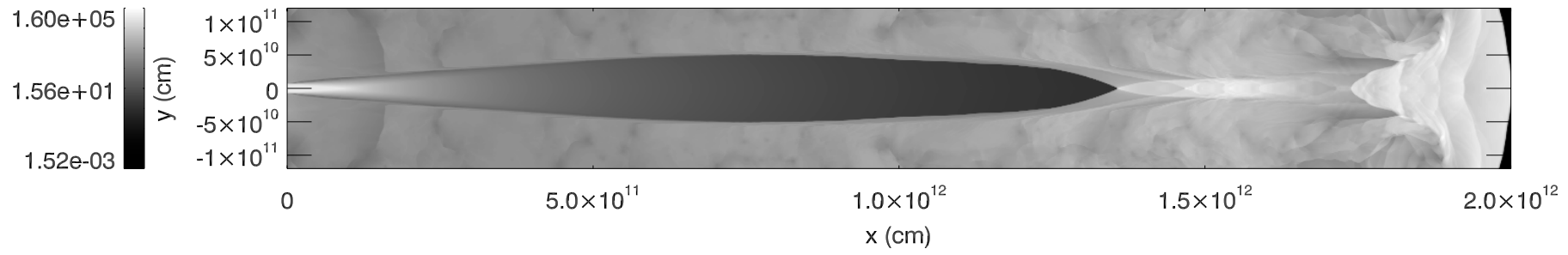

Fig. 4. Maps of axial velocity $(\mathrm{cm} / \mathrm{s})$, rest mass density $\left(\mathrm{g} \mathrm{cm}^{-3}\right)$ and pressure $(\mathrm{dyn})$ at the end of the simulation $\left(t_{\mathrm{f}}=212 \mathrm{~s}\right)$, for a powerful jet (case III). The horizontal and vertical coordinates, and the star location, are like in Fig. 1.

in the grid, and the pressure of the ambient medium $\left(P_{\text {ext }}\right)$. From Eq. (1):

$z_{\mathrm{s}}>\left(\frac{P_{\mathrm{j}, 0}}{P_{\text {ext }}}\right)^{3 / 10} z_{0}$.

Using these results, we can determine limits on the pressure (and temperature) of the jets, which are suitable to develop recollimation shocks. We fix the right-hand side of the inequality in Eq. (2) to be $\leq 10^{12} \mathrm{~cm}$, a safe estimate for $z_{\mathrm{s}}<R_{\mathrm{orb}}$, because we require maximal wind/jet interaction. We can then estimate the initial overpressure of the jet that results in a recollimation shock, inside a distance $z<R_{\text {orb }}$. If $z_{0}$ is taken as the injection point, i.e., $z_{0}=6 \times 10^{10} \mathrm{~cm}$, we obtain:

$1<\frac{P_{\mathrm{j}, 0}}{P_{\mathrm{ext}}} \leq 1.2 \times 10^{4}$

Equation (3) can be written in terms of the jet temperature $\left(T_{\mathrm{j}, 0}\right)$ and the jet density $\left(\rho_{\mathrm{j}, 0}\right)$ at the injection point:

$T_{\mathrm{j}, 0} \leq \frac{1.2 \times 10^{4} P_{\mathrm{ext}} m_{p}}{(\Gamma-1) \rho_{\mathrm{j}, 0} k_{\mathrm{B}}}=\frac{2.18 \times 10^{-4} P_{\mathrm{ext}}}{\rho_{\mathrm{j}, 0}} \mathrm{~K}$.

Both cylindrical and slab simulations show that, initially, the jets are embedded in their cocoons. However, as shown by the slabjet simulations, the stellar wind quickly starts to affect dynamically the bow-shock and the jet itself. As soon as the jet head has left the binary system, shocked wind will substitute cocoon material. We note that the wind properties change via shocking with the jet. In this case, the relative pressure of the jet with respect to the ambient would also change, at least on the side where the wind impacts. This asymmetry in the ambient medium can give rise to asymmetric shocks, generated on one side of the jet and propagating diagonally through it, as observed in the slab simulations (Figs. 1 and 3). In short, these two different scenarios, i.e. interaction with the cocoon and the wind, have to be taken into account.

When the pressure of the ambient medium corresponds to that of the cocoon, the following equation (Begelman \& Cioffi $1989)$ estimates the pressure of the latter $\left(P_{\mathrm{c}}\right)$ as a function of the jet kinetic power, advance speed of the bow shock, and the surface of interaction between the bow shock and the ambient medium $\left(A_{\mathrm{c}}\right)$ :

$P_{\mathrm{c}} \sim \frac{L_{\mathrm{j}}}{V_{\mathrm{bs}} A_{\mathrm{c}}}$

When the external pressure is exerted by the wind on the jet, it can be computed by assuming that the density of the shocked wind is four times the original density of the wind, based on the Rankine-Hugoniot jump conditions, and that most of the kinetic energy of the wind is thermalized and, thus, its internal energy is similar to the original kinetic one.

\section{Jet/Cocoon interaction}

For a typical jet with kinetic power $10^{36} \mathrm{erg} \mathrm{s}^{-1}$, bow-shock advance speed $0.1 c$, and bow-shock radius of about $3 \times 10^{11} \mathrm{~cm}$, the cocoon pressure is $P_{\mathrm{c}} \sim 10^{3} \mathrm{erg} \mathrm{cm}^{-3}$. Then, if the density in the jet at injection $\left(z_{0}=6 \times 10^{10} \mathrm{~cm}\right)$ is $\rho_{\mathrm{j}, 0} \sim 10^{-15} \mathrm{~g} \mathrm{~cm}^{-3}$, the 
temperature must be:

$$
\begin{array}{r}
T_{\mathrm{j}, 0}<3 \times 10^{14} \cdot\left(\frac{L_{\mathrm{j}}}{10^{36} \mathrm{erg} \mathrm{s}^{-1}}\right) \cdot\left(\frac{3 \times 10^{9} \mathrm{~cm} \mathrm{~s}^{-1}}{V_{\mathrm{bs}}}\right) . \\
\left(\frac{3 \times 10^{11} \mathrm{~cm}}{R_{\mathrm{c}}}\right)^{2} \cdot\left(\frac{10^{-15} \mathrm{~g} \mathrm{~cm}^{-3}}{\rho_{\mathrm{j}, 0}}\right) \mathrm{K},
\end{array}
$$

which is fulfilled accounting for the fact that the jet must be supersonic (i.e. $\left.T_{\mathrm{j}, 0}<(\Gamma-1) m_{\mathrm{p}} c^{2} / k_{\mathrm{B}} \sim 10^{12} \mathrm{~K}\right)$.

The pressure at $z_{0}$ can be set to be larger than that of the ambient medium in order to allow for the initial expansion of the jet (see Eq. (3)). A lower limit on the temperature of the jet can be fixed: $T_{\mathrm{j}, 0}>T_{\mathrm{j}, 0, \max } /\left(1.2 \times 10^{4}\right) \mathrm{K}$. In the cylindrical simulations of cases I and II, the temperatures of the jets are below this limit (see Table 2), so that they do not generate recollimation shocks (see Fig. 2). The location of the injection point in the numerical grid, $z_{0}$, is, however, arbitrary. At some point closer to the compact object, the pressure inside the jet will become larger than inside the cocoon. This is implied by the fact that the jet pressure increases towards the compact object such as $z^{5 \Gamma / 3}$. The conditions for the generation of a recollimation shock in the simulations, would then exist.

\section{Jet/wind interaction}

In the case of a jet interacting with the stellar wind, the pressure is derived as explained above to obtain $P_{\mathrm{w}} \sim 10^{2} \mathrm{erg} \mathrm{cm}^{-3}$. Using the jet density given above for the jet,

$$
\begin{aligned}
T_{\mathrm{j}, 0}<3 \times 10^{13} \cdot\left(\frac{\rho_{\mathrm{w}}}{2.8 \times 10^{-15} \mathrm{~g} \mathrm{~cm}^{-3}}\right) & \cdot\left(\frac{V_{\mathrm{w}}}{2 \times 10^{8} \mathrm{~cm} \mathrm{~s}^{-1}}\right)^{2} . \\
& \left(\frac{10^{-15} \mathrm{~g} \mathrm{~cm}^{-3}}{\rho_{\mathrm{j}, 0}}\right) \mathrm{K},
\end{aligned}
$$

which is achieved by taking into account that the jet must be supersonic.

We conclude that the presence of standing shocks within the binary system, is probable within our prediction limits.

\subsection{Radiative processes}

As discussed above, strong shocks take place in the jet head, i.e. the bow/reverse shocks, and in the jet/cocoon contact surface, i.e. the recollimation shocks. In practice, the capability of the jet head structures to produce non-thermal emission, is linked to the time that they remain within the system, because the shock-energy transfer diminishes further out, as the wind density rapidly decreases. In addition, quasi-permanent recollimation shocks between the jet and the stellar wind can also occur, as noted in Sect. 5.1. Since all these shocks are strong, and we know approximately the speed of the shocks from the simulations, we can estimate the particle-acceleration efficiency. In the following, we compute the maximum energy, and the emission spectral-energy-distribution of particles accelerated under shock conditions, similar to those shown by the simulations.

\subsubsection{Non-thermal particle production}

\section{Particle acceleration}

As a first order approach, and provided that the shock Lorentz factors are $\sim 1$, we compute the particle acceleration rate in the context of the non-relativistic (first-order Fermi) diffusive shock acceleration mechanism, in the tes-particle approximation, onedimensional case, and with a dynamically negligible magnetic field. This mechanism typically predicts, for strong shocks, a power-law particle distribution of index -2 (e.g. Drury 1983). The acceleration rate in cgs units can be computed using:

$\dot{E}=\eta q B c$,

where $\dot{E}$ is the particle energy gain per time unit, $q$ the particle charge, $B$ the magnetic field in the accelerator, and $\eta$ the acceleration efficiency coefficient, which can be calculated using the formula (e.g. Protheroe 1999):

$\eta=\frac{3}{20 K_{\mathrm{d}}}\left(\frac{V_{\mathrm{S}}}{c}\right)^{2}$

for a parallel magnetic field, where $K_{\mathrm{d}}$ is proportional to the diffusion coefficient ( $K_{\mathrm{d}}=1$ corresponds to the Bohm regime), and $V_{\mathrm{S}}$ is the speed of the shock in the upstream reference-frame. The value of the second parameter can be determined using the results of the simulations, allowing $\eta$ to be estimated. For the reverse and forward shocks, we assume velocities that are typical for when the jet head is within the binary system, which implies a value $V_{\mathrm{S}} \sim 10^{10} \mathrm{~cm} \mathrm{~s}^{-1}$. In case of recollimation shocks, as shown in Fig. 4, velocity jumps could reach similar values. In the Bohm diffusion case, $\eta \sim 2 \times 10^{-2}$. We recall that the recollimation shock shown in Fig. 4 is related to the cocoon, although such structure could also be produced by interaction of the jet lateral surface with the stellar wind.

Concerning the $B$-strength, the hydrodynamical assumption imposes an energy-density for the adopted magnetic field « the (internal plus kinetic) matter energy-density. Thus, the value assumed for the $B$-field must be well below that required for equipartition with jet matter. $B$ should be $\ll 10^{2}-10^{4} \mathrm{G}$, depending on the jet power and shock properties. This values stand for those of the (internal plus kinetic) matter energy-density in shocked material, as provided by simulations, which ranges in value between $\simeq 10^{2}-10^{6} \mathrm{erg} \mathrm{cm}^{-3}$.

We can compute the maximum energy of the particles equating Eq. (8) to the particle energy losses.

In the case of electrons, when the synchrotron energy-losses are dominant, the maximum particle energy can be derived using the equation:

$E_{\max } \approx 50 \sqrt{\frac{\eta}{B}} \mathrm{TeV}$

For inverse Compton (IC) losses in the Thomson regime $(E \ll$ $\left.5.1 \times 10^{5} \mathrm{eV} / \epsilon_{0} ; \epsilon_{0}=10 \mathrm{eV}\right)$, the maximum particle energy, after taking account of stellar radiation density in the jet, $U_{*} \approx$ $300 \mathrm{erg} \mathrm{cm}^{-3}$, can be estimated using the expression:

$E_{\max } \approx \sqrt{B \eta} \mathrm{TeV}$.

In the Klein-Nishina $(\mathrm{KN})$ regime $\left(E>5.1 \times 10^{5} \mathrm{eV} / \epsilon_{0}\right)$ (Khangulyan, et al. 2008), this expression is replaced by the following expression,

$E_{\max } \approx 10^{9}(B \eta)^{3.3} \mathrm{TeV}$

$E_{\max }$ is taken to be the lowest value of all shown $E_{\max }$. For $K_{\mathrm{d}}=1, \eta=2 \times 10^{-2}$, synchrotron energy losses are dominant for $B \gtrsim 0.2 \mathrm{G}$. $E_{\max }$ can reach $1 \mathrm{TeV}$ for $B=50 \mathrm{G}$, and $10 \mathrm{TeV}$ for $B=0.4 \mathrm{G}$.

In the case of inefficient energy losses, as it commonly happens for protons, the size of the accelerator, approximated to be the diameter of the jet at the shock location $\left(D_{\mathrm{j}, \mathrm{S}}\right)$, provides an 
upper-limit to the maximum particle energy, when equated to the particle mean-free-path (in our case, the gyroradius):

$D_{\mathrm{j}, \mathrm{S}}=r_{\mathrm{g}}=\frac{E_{\mathrm{max}}}{q B}$.

Higher energy particles escape the accelerator because they cannot be confined by the magnetic field. For $D_{\mathrm{j}, \mathrm{S}} \sim 10^{11} \mathrm{~cm}$, $E_{\text {max }}$ may reach $\sim 10^{3} \mathrm{TeV}$.

\section{Characterizing the particle energy distribution}

As soon as electrons are injected into the emitter, they lose energy via synchrotron and IC processes. Relativistic Bremsstrahlung, and ionization losses of electrons, are negligible due respectively to the long timescales, and the high ionization degree of the shocked material. Protons lose energy mainly via proton-proton collisions, although in general, the efficiency of this process is small. All particles are convected, on timescales that are shorter than the radiation timescale for the electrons with the higher energies, and protons of any energy. The convection timescale can be estimated using $\tau_{\text {conv }} \sim l_{\mathrm{d}} / V_{\mathrm{d}} \approx 400 \mathrm{~s}$, where $l_{\mathrm{d}} \approx 10^{12} \mathrm{~cm}$ and $V_{\mathrm{d}} \approx V_{\mathrm{S}} / 4$ (from the strong shock-jump conditions), are the length of the downstream region and the convection velocity, respectively (as derived from the simulations).

To respect the restriction of both a cold jet and test particle shocks, we assume that the non-thermal particle energy-density is smaller than the thermal energy-density, and fix the ratio between these energy-densities to be 0.1 close to the shock surface. Taking into account the above assumption, and the fact that downstream, thermal particles carry a power similar to that of the jet, the injected luminosity in the form of non-thermal particles is $\sim 0.1 \times L_{\mathrm{j}}$.

\subsubsection{Radiation}

\section{Leptonic emission}

We compute the SED of the synchrotron and IC components, adopting a homogeneous model for the emitting region. Because we are unable to determine the magnetic field, two $B$-values, rendering different but representative situations, have been adopted: $B=0.4$ and $50 \mathrm{G}$. The first value yields a dominant, high-energy IC component, reaching energies well above $1 \mathrm{TeV}$. The second value corresponds to a high magnetic field, in the limits of the hydrodynamical approximation. This latter case implies dominant synchrotron radiation. To compute the impact of synchrotron self-absorption, $D_{\mathrm{j}, \mathrm{S}}$, has been adopted as the width of the emitting region.

For $B=50 \mathrm{G}, U_{*} \approx 300 \mathrm{erg} \mathrm{cm}^{-3}$, and $\eta \sim 2 \times 10^{-2}$, synchrotron energy losses limit $E_{\max } \sim 1 \mathrm{TeV}$, and the energy in non-thermal particles are radiated with similar luminosities via synchrotron and IC channels due to comparable timescales. Above the injection energy, the (cooled) particle spectrum follows a power-law of exponent -2 up to the energy at which convection and radiative timescales become equal, around $1 \mathrm{GeV}$. In the one-zone approximation, electrons below $1 \mathrm{GeV}$ escape the emitter before cooling completely, although they could still produce radiation outside the binary system. Above this energy, the particle spectrum becomes radiatively cooled with index -3 .

We have also explored the case of $B=0.4 \mathrm{G}$, for which the maximum energy reaches around $10 \mathrm{TeV}$. In this case, low magnetic fields render a synchrotron component with luminosities well below the IC ones and a hard particle spectrum at the highest energies due to the impact of KN IC losses, which harden the particle energy distribution when dominant. In contrast, synchrotron and Thomson IC losses, steepen the spectrum;

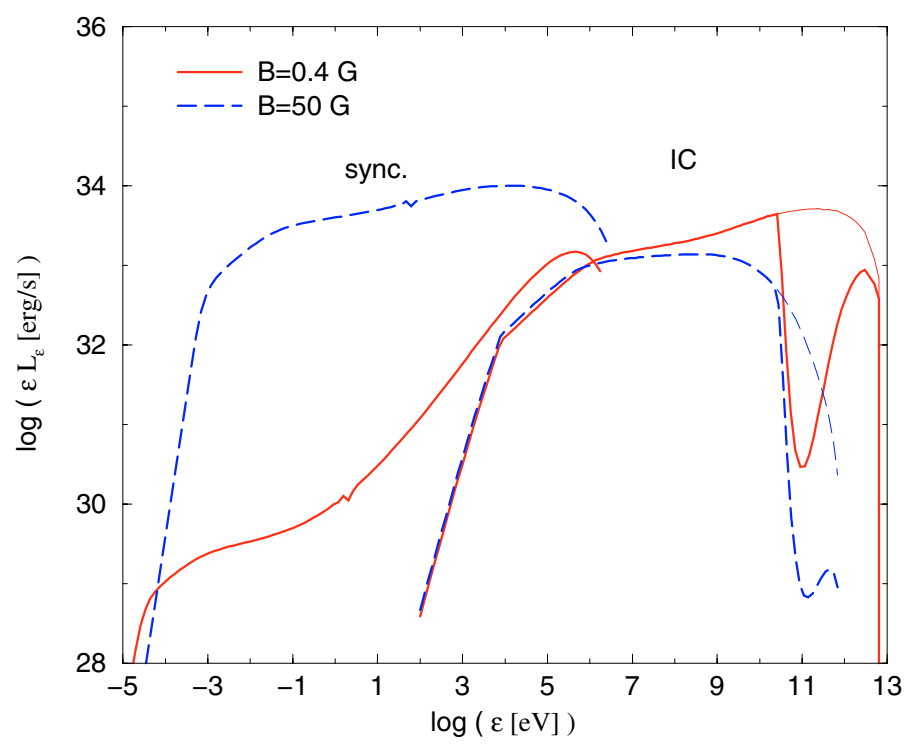

Fig. 5. Typical computed SED of the synchrotron and IC emission produced in a shock region. We adopt $B=0.4$ (solid line) and $50 \mathrm{G}$ (longdashed line). The production (thin line) and the photon-photon absorbed (thick line) SED cases are presented.

see Khangulyan et al. (2008) for a thorough discussion). In Fig. 5 we show the corresponding SED for these two cases. The total radiated luminosity is $\sim 10^{35} \mathrm{erg} \mathrm{s}^{-1}$, which is shared between the synchrotron and IC components, with the relation proportions depending on $B$. We note that the age of the source producing the SED, presented in Fig. 5, was fixed to be the typical lifetime of the shocks $\left(\sim R_{\text {orb }} / V_{\mathrm{j}}\right)$.

We note that for $B=50 \mathrm{G}$, when synchrotron emission is high, the synchrotron self-absorption frequency is $\sim 60 \mathrm{GHz}$ when assuming a low energy cutoff at $\sim m_{\mathrm{e}} c^{2}$, effectively yielding little radiation in the radio band. If the cutoff were at higher energies, electrons would radiate with a monoenergetic particle distribution in the radio band for a such magnetic field, rendering a hard SED (of exponent 4/3) and little radiation in the radio band. In this scenario, it is unclear whether radio emission from the accelerator/emitter would be detectable. Electron cooling, and transport within the flow bulk, can yield extended synchrotron radiation in the radio band, in cases where radioemitting electrons are carried to (farther) regions of appropriate $B$.

IC scattering and photon-photon absorption in the stellar radiation field have been considered using angular-averaged cross-sections. Since we do not apply this model to any specific object at present, we do not account for the geometry of these interactions. Accounting for the geometry of photonphoton absorption, the latter can have less impact for certain accelerator/emitter located locations $\left(\gtrsim 10^{12} \mathrm{~cm}\right)$ and orbital phases. This would improve the probability of detection for this kind of sources in the TeV range. We refer to Khangulyan et al. (2008) for an exhaustive discussion of IC and photonphoton absorption angular effects. Secondary pairs produced in the system may also be relevant from the radiative point of view (see Khangulyan et al. 2008; Bosch-Ramon, et al. 2008).

\section{Hadronic emission}

In the case of protons, the densities in the jet are, in general, low and proton-proton energy losses $s^{5}$ are not important, yielding a

\footnotetext{
5 Other hadronic channels, like synchrotron proton, photodisintegration, or photo-meson production, have little efficiency or an extremely high threshold energy. They are not considered here.
} 
small amount of $\pi^{0}$-decay gamma-rays. The most suitable case occurs when the proton escape velocity is low and density high in the region. This can occur in the shell region. Recollimation shocks can lead to jet-density enhancement and low-convection velocities. The mixing of jet and stellar-wind material can also lead to high-density regions that suffer the impact of relativistic protons.

In our context, gamma-ray fluxes $\gtrsim 10^{32} \mathrm{erg} \mathrm{s}^{-1}$ would be expected from proton-proton interactions if $n_{\mathrm{t}} / V_{\mathrm{d}} \gtrsim 10$ (i.e. $V_{\mathrm{d}} \sim 10^{9} \mathrm{~cm} \mathrm{~s}^{-1}$ implies $n_{\mathrm{t}} \gtrsim 10^{10} \mathrm{~cm}^{-3}$ ) for $L_{\mathrm{p}}=10^{35} \mathrm{erg} \mathrm{s}^{-1}$, where $L_{\mathrm{p}}$ and $n_{\mathrm{t}}$ are the relativistic proton luminosity and the target density, respectively. Although the efficiency of hadronic processes is relatively low for the production of gamma-rays, the interaction of powerful jets and dense winds could indeed lead to the generation of detectable $\mathrm{TeV}$ emission. In these proton-proton interactions, neutrinos (e.g. Romero et al. 2005; Aharonian et al. 2006a; Christiansen, et al. 2006) and secondary electron-positron pairs would also be produced, with luminosities similar to the gamma-ray ones. The radiation of secondary pairs would be relevant mainly at low energies (e.g. Orellana et al. 2007).

\section{Variability}

Initially, the forward and reverse shocks can produce nonthermal radiating particles as long as the jet ttavels through the system, with timescales $\sim R_{\text {orb }} / V_{\mathrm{j}} \approx 300 \mathrm{~s}$. In the case where recollimation shocks in the jet, due to cocoon material pressure, form, particle acceleration lasts as long as the cocoon pressure is higher than the pressure of the jet.

Because the cocoon pressure is roughly proportional to $\propto 1 / t^{2}$, as soon as the jet head is outside the binary system, cocoon recollimation-shocks, inside the system region, should not last much longer than $\sim R_{\mathrm{orb}} / V_{\mathrm{j}}$. All such events should appear as short bursts of $\mathrm{X}$-rays, and possibly also $\mathrm{GeV}-\mathrm{TeV}$ and radio band emission.

Irregular jet-ejection on timescales longer than $R_{\text {orb }} / V_{\mathrm{w}}$ (the wind replenishment timescale), in a massive system, could lead to high-energy, recurrent flares, as observed for Cygnus X-1 (Albert et al. 2007), and in some orbital phases of LS 5039 (Aharonian et al. 2006b), and LS I +61 303 (Rico 2007). The observed hour timescale variability associated with these flares, could be explained by a new ejection facing stellar-wind, replenished during a previous jet inactivity period of similar or longer duration. Eventual recollimation shocks due to wind ram pressure, instead of cocoon pressure, could stand as long as the jet injection lasts and the wind properties are relatively smooth. The variability of emission in this phase would be likely dominated by jet injection fluctuations or wind inhomogeneities, with associated timescales $\gtrsim R_{\mathrm{j}} / c$.

\subsection{The fate of the jet}

As a test for jet bending, numerical simulations in slab geometry have been performed. It has been shown that the wind can efficiently transport shell and cocoon material sidewards. In the same way, the wind can significantly deflect the supersonic jet itself via transferring of enough lateral momentum. We have shown that even in the case of a jet with kinetic power $10^{36} \mathrm{erg} \mathrm{s}^{-1}$, the wind appears to be able to disrupt the flow. Such a disrupted jet can look like a radio structure changing its morphology along the orbit, such as that found by Dhawan et al. (2006) in LS I +61 303, and interpreted by these authors as the collision between the star and pulsar winds. This interpretation would be challenged by the morphologies obtained from hydrodynamical simulations by Romero et al. (2007b) and Bogovalov et al. (2007).

The simulations and calculations presented here show that the recollimation shocks can, on the one hand, develop easily inside the binary-system region and, on the other hand, influence significantly the evolution of the jet. As already stated, these shocks can both generate radiation efficiently and favor jet mass-loading and deceleration via pinching, which makes winddeflection easier. These shocks have already been reported in a work by Peter \& Eichler (1995), which studied the collimation of jets by the inertia of the ambient medium. In the maps shown in that work, shocks similar to those observed in Fig. 4, were detected.

The jet parameters that we have adopted produce small growth-rates of Kelvin-Helmholtz instability, mainly in the cases II and III. Therefore, disruption due to the growth of such an instability, inside the region of interaction with the wind, is not expected. Otherwise, our simulations show that the jets develop non-linear structures due to their initial overpressure, thus the growth of linear instabilities is not expected either. In short, the stability of the jet depends mainly on its inertia, in contrast to the inertia of the wind, and on the strength of the recollimation shocks (Perucho et al. 2005, 2007).

\section{Summary}

The crossing of a hydrodynamical supersonic jet, through the inner parts of an HMMQ, renders strong forward/reverse and recollimation shocks. These shocks produce strong pressure and density enhancements, and some jet deceleration. The impact of the wind ram pressure on weak jets can prevent the formation of larger scale jets because of strong bending and jet disruption. We estimate that this occurs for jet kinetic luminosities below $10^{36} \mathrm{erg} \mathrm{s}^{-1}$ for the strong winds of O-type stars.

If efficient leptonic and/or hadronic particle acceleration takes place, the presence of photon, matter and magnetic fields, will yield significant amounts of radiation from radio to very high energies. On the one hand, leptons would radiate mainly via synchrotron and IC processes. On the other hand, protons could interact with shell nuclei producing gamma-rays via neutral pion decay, and electron-positron pairs and neutrinos via decay of charged pions. Secondary pairs may also yield some amount of low-energy radiation. In addition, the complexity of the wind/jet interactions leads to a plethora of variability timescales of the emission that could be more relevant than any intrinsic jet fluctuation.

Further three-dimensional numerical simulations including more realistic implementations of the problem, magnetic fields, and radiative processes, should improve our understanding of the physics of jets in microquasars.

Acknowledgements. The authors benefited from valuable discussions with D. Khangulyan. The authors thank G. E. Romero for a thorough reading of the manuscript. V.B-R. acknowledges support by DGI of MEC under grant AYA2007-68034-C03-01 and FEDER funds. V.B-R. gratefully acknowledges support from the Alexander von Humboldt Foundation. This work was supported in part by the Spanish Dirección General de Enseñanza Superior under grant AYA2004-08067-C03-01. M.P. acknowledges support from a postdoctoral fellowship of the Generalitat Valenciana (Beca Postdoctoral d'Excel-lència).

\section{References}

Aharonian, F. A., \& Atoyan, A. M. 1996, SSRv, 75, 357

Aharonian, F. A., \& Atoyan, A. M. 1996, NewAR, 42, 579

Aharonian, F. A., Anchordoqui, L. A., Khangulyan, D., \& Montaruli, T. 2006a, J. Phys. Conf. Ser., 39, 408 
Aharonian, F., Akhperjanian, A. G., Bazer-Bachi, A. R., et al. 2006b, A\&A, 460, 743

Albert, J., Aliu, E., Anderhub, H., et al. 2007, ApJ, 665, L51

Aloy, M. A., Ibáñez, J. M., Martí, J. M., Gómez, J. L., \& Müller, E. 1999, ApJ, 523, L125

Araudo, A. T., Romero, G. E., Bosch-Ramon, V., \& Paredes, J. M. 2007, A\&A, 476, 1289

Begelman, M. C., \& Cioffi, D. F. 1989, ApJ, 345, L21

Begelman, M. C., Blandford, M. D., \& Rees, M. J. 1984, RvMP, 56, 225

Bell, A. R. 1978 MNRAS, 182, 443

Bogovalov, S. V., Khangulyan, D., Koldoba, A. V., Ustyugova, G. V., \& Aharonian F. A. 2007, MNRAS, submitted [arXiv: astro-ph/0710.1961]

Bosch-Ramon, V., Aharonian, F. A., \& Paredes, J. M. 2005, A\&A, 432, 609

Bosch-Ramon, V., khnagulyan, D., \& Aharonian, F. A. 2008, A\&A, 482, 397

Bykov, A. M., \& Fleishman, G. D. 1992, MNRAS, 255, 269

Casares, J., Ribó, M., Ribas, I., Paredes, J. M., Martí, J., \& Herrero, A. 2005, MNRAS, 364, 899

Corbel, S., Fender, R. P., \& Tzioumis, A. K., et al. 2002, Science, 298, 196

Corbel, S., Kaaret, P., \& Fender, R. P. 2005, et al. ApJ, 632, 504

Christiansen, H. R., Orellana, M., \& Romero, G. E. 2006, Phys. Rev. D, 73, 3012 Derishev, E. V., Aharonian, F. A., Kocharovsky, V. V., \& Kocharovsky, V1. V. 2003, Phys. Rev. D, 68, 3003

Dhawan, V., Mioduszewski, A., \& Rupen, M. 2006, in Proc. of the VI Microquasar Workshop, Como-2006

Drury, L. 1983, RPPh, 46, 973

Fermi, E. 1949, Phys. Rev., 75, 1169

Gabici, S., \& Blasi, P. 2004, APh, 20, 579

Gallo, E., Fender, R., \& Kaiser, C., et al. 2005, Nature, 436, 819

Gies, D. R. \& Bolton, C. T. 1986, ApJ, 304, 371

Gómez, J. L. 2001, Ap\&SS, 276, 281

Hardee, P. E., \& Hughes, P. A. 2003, ApJ, 583, 116

Heindl, W. A., Tomsick, J. A., Wijnands, R., \& Smith, D. M. 2003, ApJ, 588, L97

Heinz, S. 2002, A\&A, 388, L40

Heinz, S. 2006, ApJ, 636, 316

Heinz, S., \& Sunyaev, R. 2002, A\&A, 390, 751

Kang, H., Ryu, D., \& Jones, T. W. 1996, ApJ, 56, 422

Khangulyan, D., Aharonian, F. A., \& Bosch-Ramon, V. 2008, MNRAS, 383, 467

Komissarov, S. S., \& Falle, S. A. E. G. 1998, MNRAS, 297, 1087

Krause, M. 2005, A\&A, 431, 45

van der Laan, H. 1962, MNRAS, 124, 179
Leahy, J. P. 1991, in Beams and Jets in Astrophysics, ed. P. A. Hughe (Cambridge: Astrophysics Series), 100

Leismann, T., Antón, L., Aloy, M. A., et al. 2005, A\&A, 436, 503

Marshall, H. L., Canizares, C. R., \& Schulz, N. S. 2002, ApJ, 564, 941

Martí, J., Rodriguez, L. F., Mirabel, I. F., \& Paredes, J. M. 1996, A\&A, 306, 449

Martí, J. M., Müller, E., Font, J. A., Ibáñez, J. M., \& Marquina, A. 1997, ApJ, 479,151

Mirabel, I. F., \& Rodríguez, L. F. 1999, AR\&A, 37, 409

Mizuno, Y., Hardee, P. E., \& Nishikawa, K.-I. 2007, ApJ, 662, 835

Orellana, M., Bordas, P., Bosch-Ramon, V., Romero, G. E., \& Paredes, J. M. 2007, A\&A, 476, 9

Owocki, S., \& Cohen, D. 2006, ApJ, 648, 565

Paredes, J. M., Bosch-Ramon, V., \& Romero, E. 2006, A\&A, 451, 259

Paredes, J. M., Ribó, M., \& Bosch-Ramon, V., et al. 2007, ApJ, 664, L39

Perucho, M., Martí, J. M., \& Hanasz, M. 2005, A\&A, 443, 863

Perucho, M., Lobanov, A. P., Martí, J. M., \& Hardee, P. E. 2006, A\&A, 456, 493

Perucho, M. \& Martí, J. M. 2007, MNRAS, in press, [arXiv: 0709.1784]

Peter, W., \& Eichler, D. 1995, ApJ, 438, 244

Protheroe, R. J. 1999, ADP-AT-98-9 [astro-ph/9812055]

Puls, J., Markova, N., Scuderi, S., et al. 2006, A\&A, 454, 625

Rees, M. J., \& Gunn, J. E. 1974, MNRAS, 167, 1

Ribó, M. 2005, ASPC, 340, 269

Rico, J., for the MAGIC collaboration, talk presented in the conference: High energy processes in relativistic jets, Dublin, Ireland

Rieger, F. M., \& Duffy, P. 2004, ApJ 617, 155

Romero, G. E., Torres, D. F., Kaufman Bernadó, M. M., \& Mirabel, I. F. 2003, A\&A, 410, L1

Romero, G. E., \& Orellana, M. 2005, A\&A, 439, 237

Romero, G. E., Owocki S. P., Araudo, A. T., Townsend, R., \& Benaglia P. 2007a, workshop proceedings, Clumping in Hot Star Winds [arXiv: astro-ph/0708.1525]

Romero, G. E., Okazaki, A. T., Orellana, M., \& Owocki, S. P. 2007b, A\&A, 474, 15

Rosen, A., Hughes, P.A., Duncan, G.C., \& Hardee, P.E. 1999, ApJ, 516, 729

Scheck, L., Aloy, M.A., Martí, J. M., Gómez, J.L., \& Müller, E. 2002, MNRAS, 331,615

Tsinganos, K., Vlahakis, N., Bogovalov, S. V., Sauty, C., \& Trussoni, E. 2004, Ap\&SS, 293, 55

Tudose, V., Fender, R. P., \& Kaiser, C. R., et al. 2006, MNRAS, 372, 417

Velázquez, P. F., \& Raga, A. C. 2000, A\&A, 362, 780

Zealey, W. J., Dopita, M. A., \& Malin, D. F. 1980, MNRAS, 192, 731 